\title{
Lateral transport of traffic pollutants in complex urban area
}

\author{
Zuzana Kluková ${ }^{1,2, *}$, Štěpán Nosek $^{1}$, Zbyněk Jaňour ${ }^{1}$, and Libor Kukačka ${ }^{2}$ \\ ${ }^{1}$ Institute of Thermomechanics, Czech Academy of Sciences \\ ${ }^{2}$ Department of Atmospheric Physics, Charles University
}

\begin{abstract}
Owing to the spatial variability of an urban area, even the transport of passive scalar is complex. The examination of the pollutant transport in such turbulent flow as occur within the urban canopy requires the measurements not only the advective but also the turbulent part of this transport. However, recent measurement techniques for the turbulent transport have their limitations. In particular, these measurements are very demanding if one needs to analyze the ventilation processes of the street through the entire streetcanyon openings and which needs to be performed also in the positions where the handling with the measurement instruments might comprise a complex issue or is even impossible. Thus we present a comparison of two methods for the assessment of turbulent and advective pollution fluxes through the lateral openings of two different street canyons with respect to the wind direction and the roof-height nonuniformity to evaluate the importance of the measurement of these fluxes nearby the street-canyon walls. Both of them are based on the simultaneous point measurement of concentration and lateral velocity component and the interpolation between the last measured point and the street-canyon borders, but in the second method the measured area is extended from $77 \%$ to $84 \%$ of the total area of the lateral openings.
\end{abstract}

\section{Introduction}

Anthropogenic pollution of the atmosphere is a very actual problem. The most discussed is the global warming as the consequence of rising concentrations of greenhouse gases (e.g. carbon dioxide) but for example the smog situations caused by high concentration of gaseous and particulate pollutants are the serious environmental issues for many megacities around the world. These smog situations have an adverse impact on human health and for this reason it is very important to examine and understand the mechanisms and processes that influence the dispersion of the pollutants within urban areas. This becomes more crucial in the case of an accidental or deliberate release of harmful substances within a local spot of a city.

To better understand the atmospheric dispersion phenomena within such complex geometry as the urban area, researchers developed three main experimental approaches: experiments conducted under the real conditions (field experiments), experiments conducted under the laboratory conditions (wind-tunnel experiments), and numerical experiments (Computational Fluid Dynamics - CFD). Each of these approaches has its own disadvantages and limitations. For instance, due to financial and logistic demands, the field experiments are recently frequently replaced with CFD models. However, these models still need to be validated against wind-tunnel or field tests [1].
The first wind-tunnel studies were focused on flow and dispersion around a single obstacle (cube e.g., [2]). This geometrically simplest 3D "generic case" has been intensively researched and nowadays is well-understood. However, recently the research is focused on more realistic and hence complex geometries to better understand the dispersion processes and hence ventilation of the real urban scenarios (see [3-4]). Compared to CFD, the wind-tunnel experiments encounter with many issues to perform the measurements of the turbulent transfer of a passive scalar even in a local point due to the restrictions of upto-date measurement techniques [5]. The issue is even more challenging if one needs to investigate the overall pollutant transport (including the turbulent) and thus the streets formed by buildings, representing the "street canyons", provide restrictions for the measurement techniques. Some previous studies $[4,5]$ suggested that there might be appreciable pollutant transport processes nearby the canyon walls and bottom, especially if the roof height is not uniform.

Therefore, this study is focused on performance assessment of two methods for investigation of the total pollutant transport of the street canyons through their entire lateral openings and hence nearby their walls and bottom in order to clarify their importance and limitations for the street-canyon ventilation pollution analysis.

*Corresponding author: klukovaz@it.cas.cz 


\section{Methods}

\subsection{The urban-area model}

The study was performed on 3D urban array model with the geometry typical for Central European cities (Fig. 1). The model of a scale of 1:400 was composed by evenly spaced (of $0.8 H$, where $H=25 \mathrm{~m}$ is the average height of the urban area at full scale) $8 \times 4$ court-yard type buildings of constant length $(4.8 H)$ and width $(2.4 H)$, and with pitched roofs. To take into account the full treedimensionality of the problem, the buildings were composed from 8 segments of the constant width and length but of different height which varied arbitrarily between $0.8 \mathrm{H}, 1 \mathrm{H}$ and $1.2 \mathrm{H}$ (see the grey contour in Fig. 1).

For this study the turbulent and advective (and hence also the total) pollution fluxes through the lateral openings of two different street canyons were investigated in detail (Fig. 1). The arrangement of the right canyon $(\mathrm{R})$ consisted of two step-up (the windward building higher than the leeward) and two uniform roofheight configurations. The arrangement of the left canyon (L) represented two step-down (the windward building lower than the leeward), one step-up and one uniform roof-height configurations.

The pollution from the traffic was simulated by a homogenous ground-level source of a passive gas (ethane) situated in the centre of the investigated street canyons. A total length of the source was $16 \mathrm{H}$ and formed by 54 equidistantly spaced tubes with an inner diameter of $0.005 \mathrm{H}$ and a length of $1.6 \mathrm{H}$ through which the ethan was emitted homogenously at the total rate $Q=18 \mathrm{ml} \mathrm{s}^{-1}$.

To assess the importance of the measurement nearby the walls, the pollution flux fields were obtained using two methods. The first method included the turbulent and advective pollution flux measurement at the basic grid (red dots in Fig. 2) and the interpolation between this grid and the street-canyon walls and bottom at the distance of $0.1 \mathrm{H}$ (thus $77 \%$ of the total lateral opening was measured). In the second method an extra measurement at half of this distance was conducted (green dots in Fig. 2), and hence the distance for the interpolation between the measured grid and the canyon borders was decreased to $0.05 H$ ( $84 \%$ of the total lateral opening was measured). We take the second method as the reference and truth-based method.
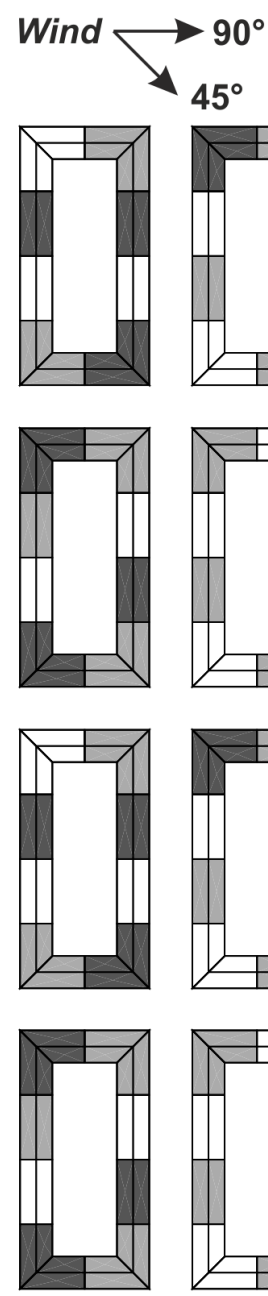
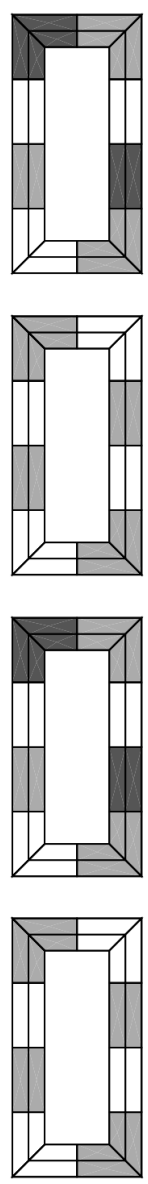
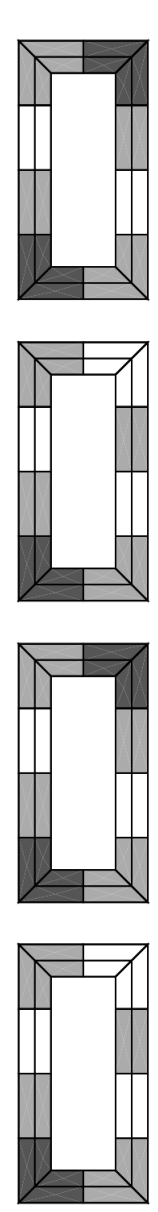

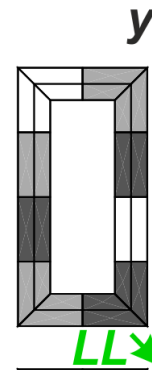

$y_{4}$
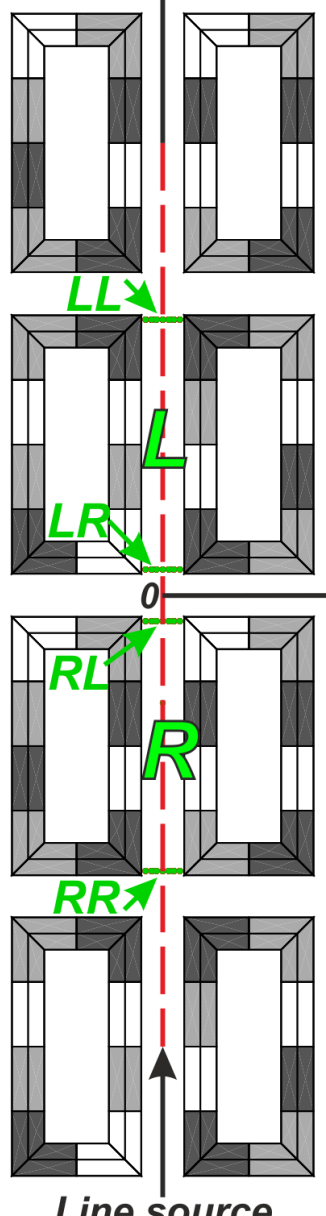

$z / H: \quad 0.8 \quad 1.0 \quad 1.2$
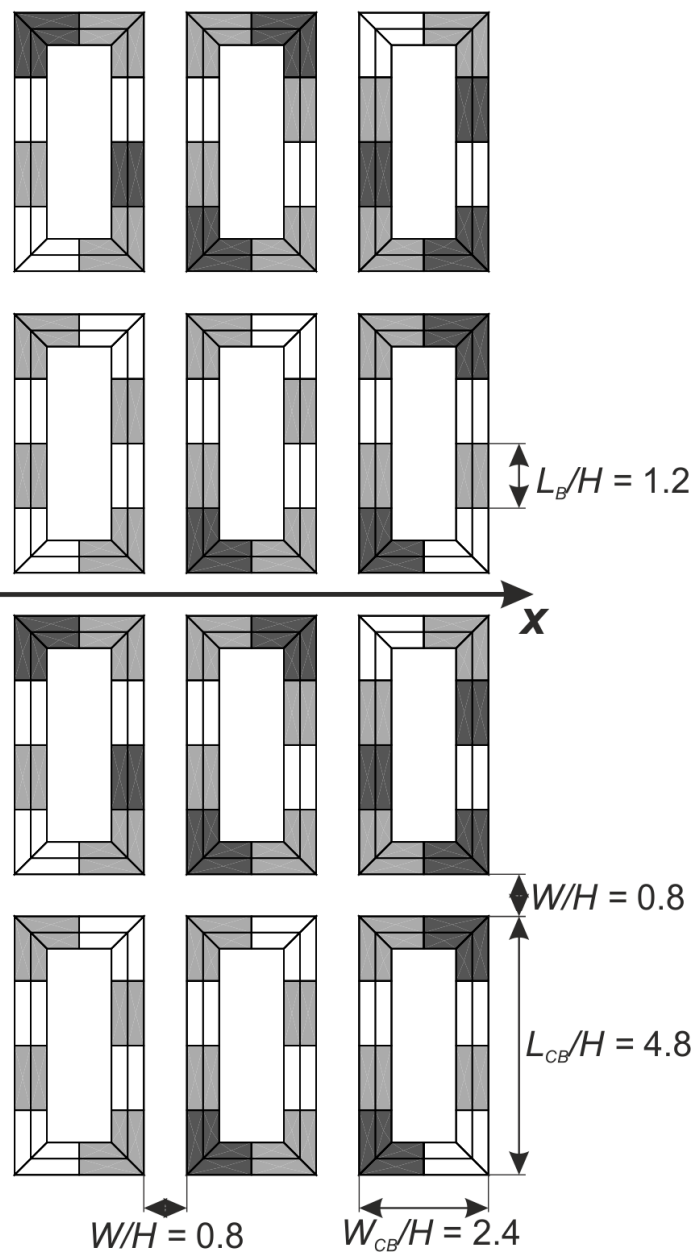

Fig. 1. Top view of the modelled urban area with the investigated lateral openings (the green arrows and labels) of the street canyons. The grey contour represents the dimensionless height $(z / H)$ of the courtyard building's segment. 


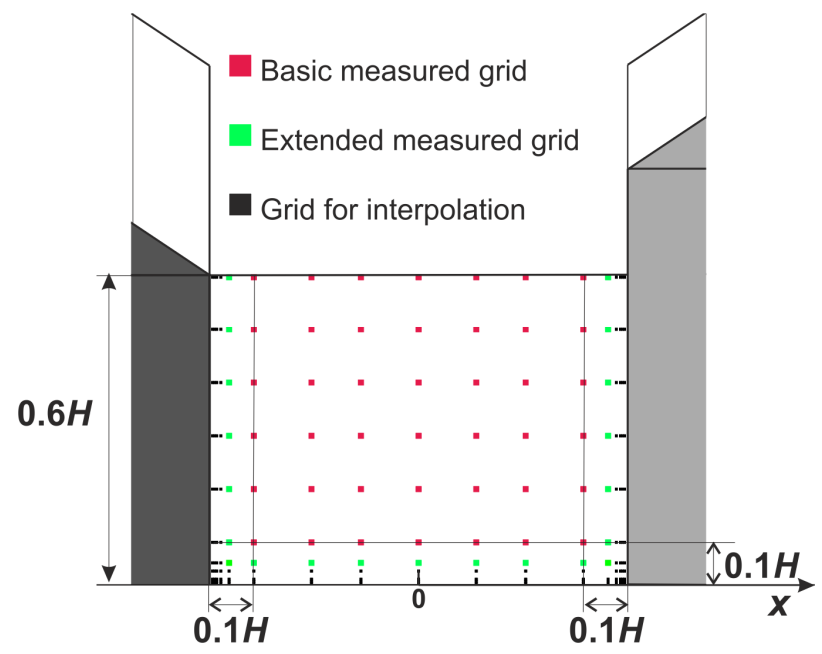

Fig. 2. Grid definition. The view is taken for the right lateral opening of the right canyon (RR) and the wind is from the left to the right for the case of the perpendicular wind direction.

\subsection{Wind tunnel}

The measurement was realized in an open wind tunnel of the Czech Academy of Sciences in Nový Knín. The cross-section dimensions of this wind-tunnel are $1.5 \times 1.5 \mathrm{~m}$ and the lenghts of the development and the test sections are $20.5 \mathrm{~m}$ and $2 \mathrm{~m}$, respectively. The wind velocity can be maintained within a range of 0.1 to $10 \mathrm{~ms}^{-1}$ using a frequency convertor of the fan. However, for this study a velocity about $U_{\text {ref }}=6 \mathrm{~ms}^{-1}$ measured by a Prandtl tube at a fixed position $(1 \mathrm{~m}$ above the windtunnel bottom and $4 \mathrm{~m}$ upwind from the model) was used in order to provide sufficiently rough turbulent flow required for proper modelling of atmospheric dispersion in urban areas [6]. In particular, the neutrally stratified turbulent boundary layer (BL) was formed by the optimal configuration of spires and roughness elements at the bottom of the wind-tunnel development section. Vertical profiles (Fig. 3, right) of the mean velocity components (longitudinal $U$ and vertical $W$ ), their turbulent characteristics (U-RMS and $W-R M S$ ) and momentum flux $\left(\left\langle u^{\prime} w^{\prime}\right\rangle\right)$ were obtained at three lateral positions just in front of the modelled urban area (Fig. 3, left) using a two-dimensional laser Doppler anemometry (LDA). The profiles were normalised by the reference velocity $U_{\text {ref }}$ and the parameters of the approaching BL for both simulated wind directions, obtained from the logarithmic law, are presented in Table $\mathbf{1}$.

Table 1. Parameters of the approaching BL

\begin{tabular}{|c|cc|}
\hline Parameter & $\mathbf{9 0}^{\circ}$ & $\mathbf{4 5}^{\circ}$ \\
\hline Roughness length $\mathrm{z}_{0}$ & $0.94 \mathrm{~m}$ & $0.87 \mathrm{~m}$ \\
Displacement height $\mathrm{d}_{0}$ & $10.3 \mathrm{~m}$ & $10.6 \mathrm{~m}$ \\
${\text { Friction velocity }{ }^{*}}^{*}$ & $0.47 \mathrm{~ms}^{-1}$ & $0.47 \mathrm{~ms}^{-1}$ \\
\hline
\end{tabular}
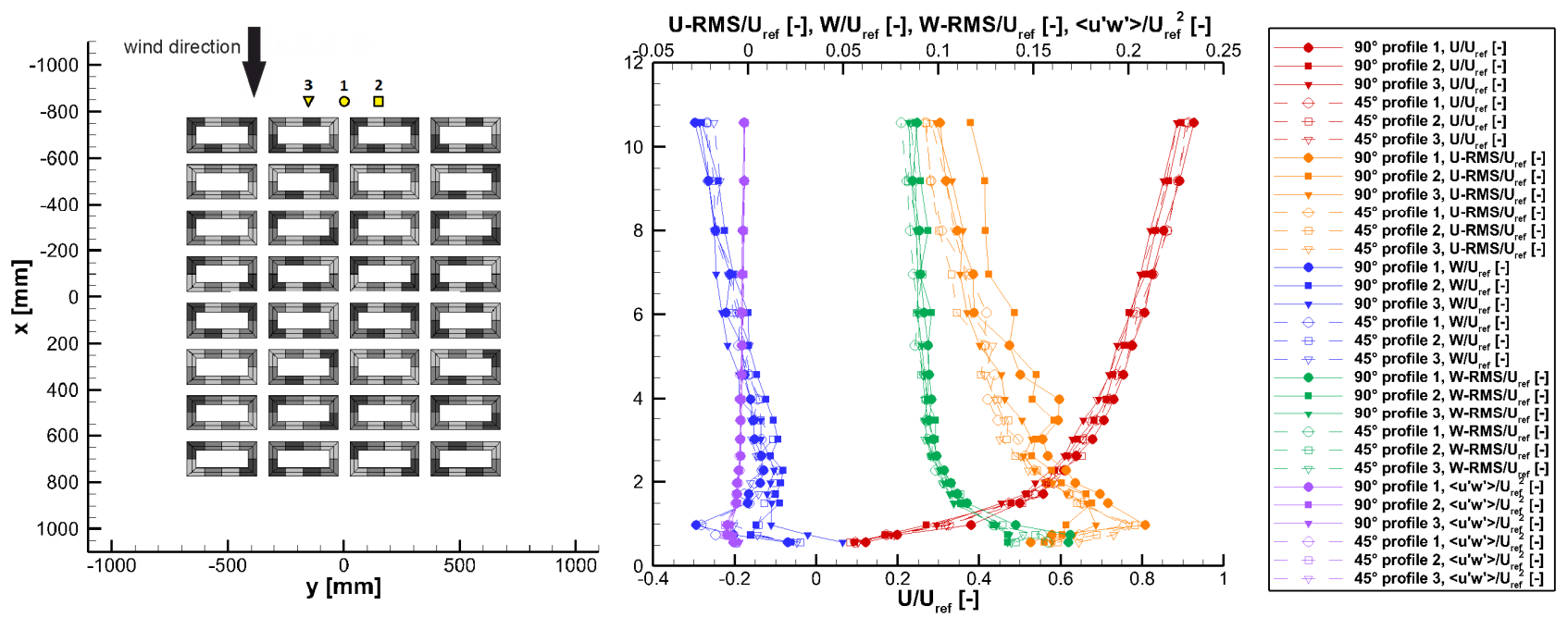

Fig. 3. Vertical profiles (right) of the mean velocities and turbulence of the approaching BL for the modelled configurations and for each of the position labelled (left). The positions were the same as for the oblique wind direction $\left(45^{\circ}\right)$. 


\subsection{Measurement techniques}

Pollution fluxes through the investigated lateral openings were examined following the method used by Nosek [4], but instead of two velocity components (longitudinal and lateral) only the lateral velocity component together with the concentration were measured approx. at the same point simultaneously using the LDA and Fast Flame Ionization Detector (FFID), respectively, for this study. As in [4], the LDA and FFID probes were mounted together on a 3D traverse system (see Fig. 4) to ensure the fixed relative position between the LDA and the FFID. The FFID tube was situated $1 \mathrm{~mm}$ above, $1 \mathrm{~mm}$ behind and $1 \mathrm{~mm}$ beside of the LDA measuring volume without a substantial influence on the LDA measurement.

The FFID sampling frequency was constant and set to $500 \mathrm{~Hz}$, whereas the LDA sampling frequency varied depending on the region from $25 \mathrm{~Hz}$ near the canyon bottom to about $1000 \mathrm{~Hz}$ at the centre of the lateral opening. Thereby, the measured 120 -s-long time series (tested as statistically independent with respect to the performed sampling frequencies) of instantaneous concentration and velocities were reconstructed according to that time series which had the shortest frequency (LDA or FFID). Owing to the length of the FFID sampling tube $(200 \mathrm{~mm})$, we tested the influence of the emerging FFID time delay relative to that of the LDA by several test analysis and found that there is negligible impact of this delay on the results of the turbulent pollution fluxes (within the uncertainty of their measurement and calculation which was estimated on $10 \%)$.

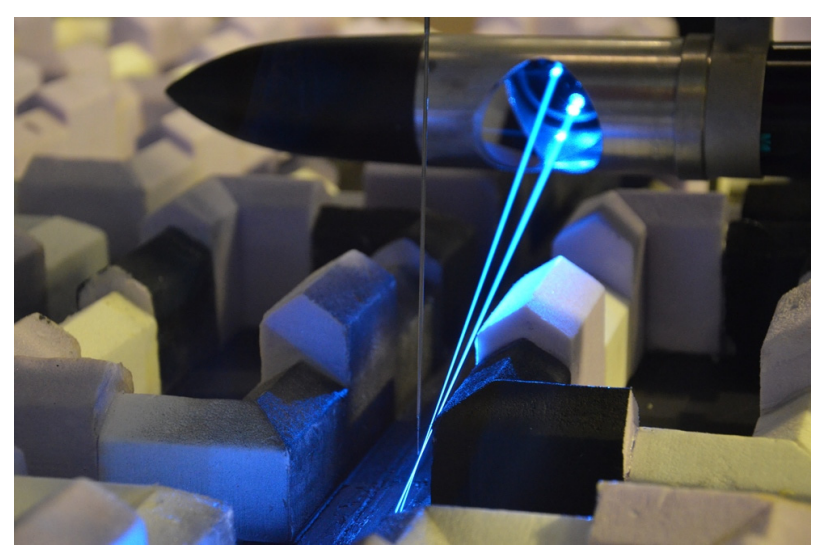

Fig. 4. The configuration of the LDA and FFID for the point measurement of the turbulent and advective pollution fluxes through the street-canyon lateral opening (the same opening as in Fig. 2).

\section{Results and discussion}

Fig. 5 and 6 present the mean dimensionless total lateral pollution flux fields $\left(\left\langle c^{*} w\right\rangle / U_{\text {ref }}\right.$, where $c^{*}$ is the measured concentration $c$ non-dimensionalized by $Q / U_{\text {ref }} H L$ and the angular brackets represent the time averaging) through the lateral openings of the right (R) and left (L) street canyons for both investigated methods and wind perpendicular to the along-canyon axis. Thereafter, in Fig. 7 and 8 are presented the same canyon opening but for the case of the oblique wind. The positive and negative values indicate for the canyon the outgoing and incoming pollution fluxes, respectively. The top of the fields was set according to the lowest roof geometry, thus at the dimensionless height $z / H=0.6$, for all investigated openings. For the interpolation we used MATLAB function inpaint_nans, based on sparse linear algebra and PDE, and for the boundary conditions (the walls and bottoms of the canyon) we set $\langle c * w\rangle / U_{\text {ref }}=0$.

From the first view it is clear that there are appreciable differences between the two investigated methods. While the interpolation between the basic grid and the canyon walls and bottom caught particular pollution flux patterns nearby the canyons' borders (left columns in Figs. 5-6), it was not able to catch the significant pollution flux gradients which were measured at extended grid for both investigated winds (at the distances shorter than $0.1 H$, right columns in Figs. 5-6). This can be more clearly seen in the case of the oblique wind where the significant pollution flux gradients (caused by the advection of the pollutant) nearby the canyons' bottoms are omitted almost completely by the interpolation (Figs. 7-8). These significant outgoing and incoming fluxes suggest that there would be a high risk of high uncertainty if one need to perform the analysis of the street-canyon ventilation from the traffic within an urban area of regular layout but of non-uniform height.

To quantify the differences between the pollution flux fields obtained from the basic grid and those from the extended grid, the total pollution flux through the particular lateral opening was computed first from the surface integral using the areas presented in Figs. 5-8 as

$$
F=\iint<c * v>/ U_{r e f} d S,
$$

and then the relative differences between the two applied methods were given as

$$
\text { Diff }=\left|F_{e}-F_{b}\right| /\left|F_{e}\right|,
$$

where $F_{b}$ and $F_{e}$ are the total pollution fluxes for the basic and the extended grid, respectively. The results of these relative differences are listed in Table 2 . The bolted values represent the differences which are higher than the overall uncertainty of the measured and computed total pollution flux fields $(10 \%)$, and hence are significant.

The results presented in Table 2 clearly show that there is only one insignificant difference (LR opening in the case of the perpendicular wind, $90^{\circ}$ ) between two applied methods. The biggest relative difference can be observed for the right opening of the left canyon (RR) and perpendicular wind $(117 \%)$. The reason for such high difference is that the total pollution flux from the first method resulted in overall incoming (Fig. 5a, left column) flux into the canyon while the second method resulted in overall outgoing (Fig. 5a, right column) flux from the canyon for that same opening. This can be also deduced from the pollution fluxes fields presented in Fig. 5 nearby the bottoms (Fig. 5a, $z / H<0.1 H$ ) where the measurements (Fig. 5a, right column) caught the 
appreciable outgoing pollution flux from the canyon in comparison with the same but interpolated area (Fig. 5a, left column) where the incoming pollution flux was computed. This confirms the preliminary conclusion stated during the qualitative comparisons of the pollution flux fields that the pollution flux measurements are needed to be performed at least at the distance $x / H=0.05$ in order to avoid high uncertainty in interpolation methods.
Table 2. The relative percentage differences between total pollution fluxes through the investigated lateral openings computed from the basic measured grid and those from the extended measured grid

\begin{tabular}{|c|cc|}
\hline Lateral opening & Wind dir. $90^{\circ}$ & Wind dir. 45 \\
\hline RR & $\mathbf{1 1 7 \%}$ & $\mathbf{1 2 \%}$ \\
RL & $\mathbf{1 8 \%}$ & $\mathbf{1 3 \%}$ \\
LR & $10 \%$ & $\mathbf{2 2 \%}$ \\
LL & $\mathbf{1 6 \%}$ & $\mathbf{2 6 \%}$ \\
\hline
\end{tabular}
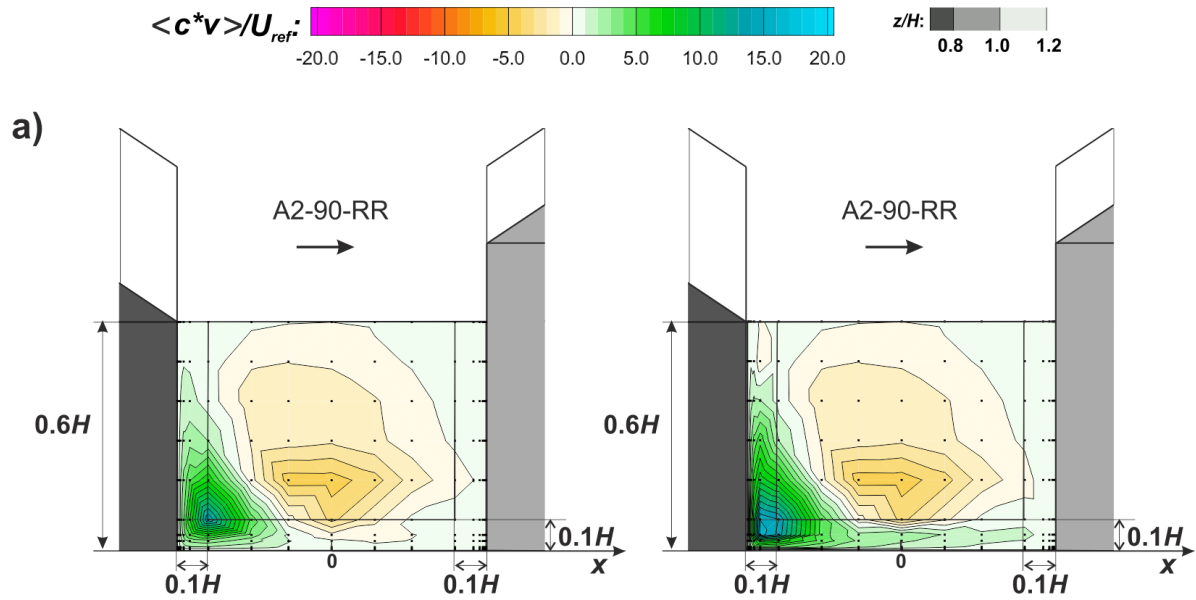

b)

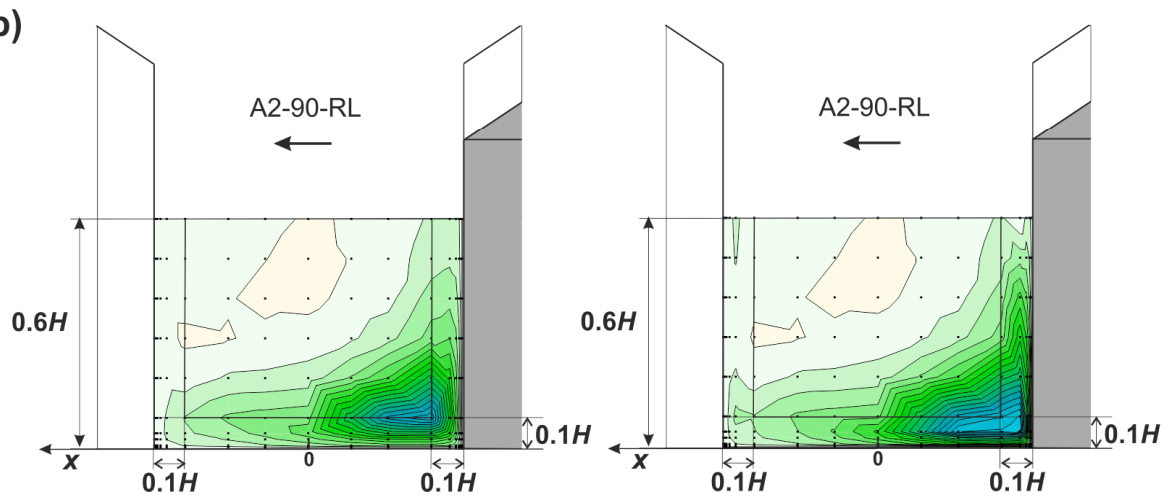

Fig. 5. Mean dimensionless total lateral pollution flux fields $\left\langle c{ }^{*} v\right\rangle / U_{\text {ref }}$ through the a) right (RR) and b) left (RL) lateral opening of the right canyon (R), computed for the perpendicular wind $\left(90^{\circ}\right)$ and from the basic (left column) and extended (right column) grid. 


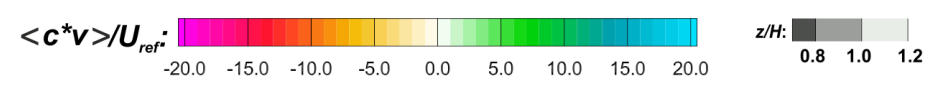

a)
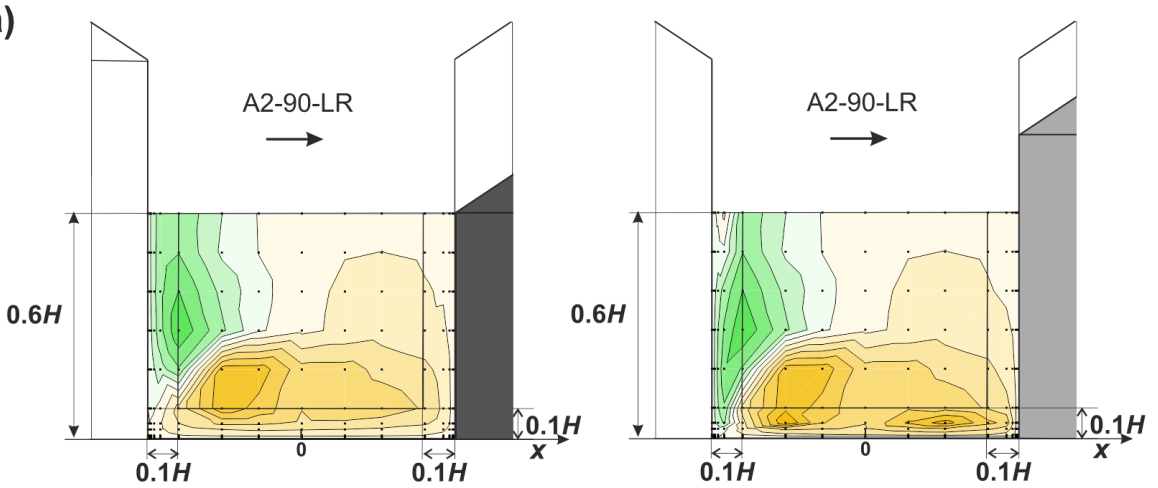

b)

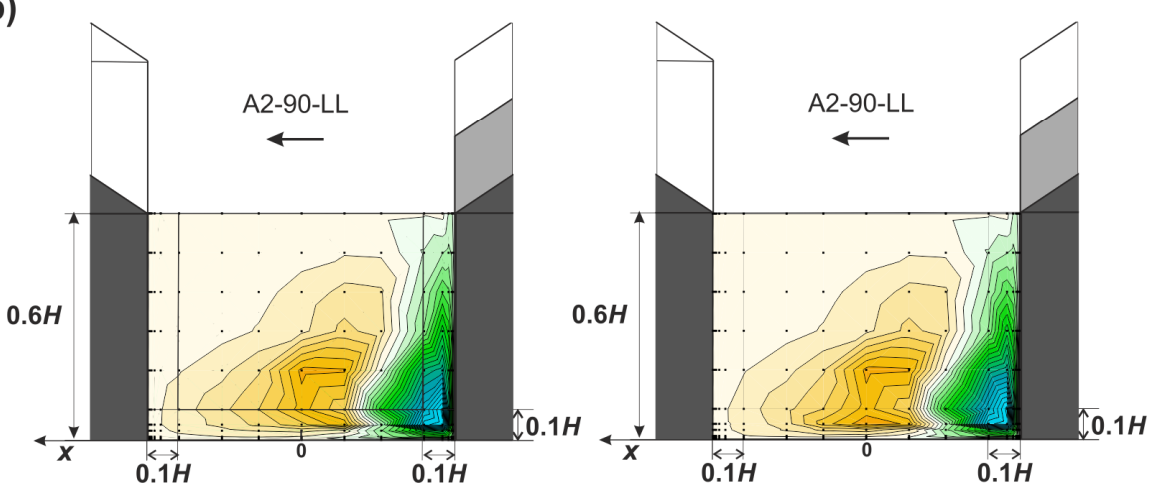

Fig. 6. The same as in Fig. 5, but for the left canyon (L).
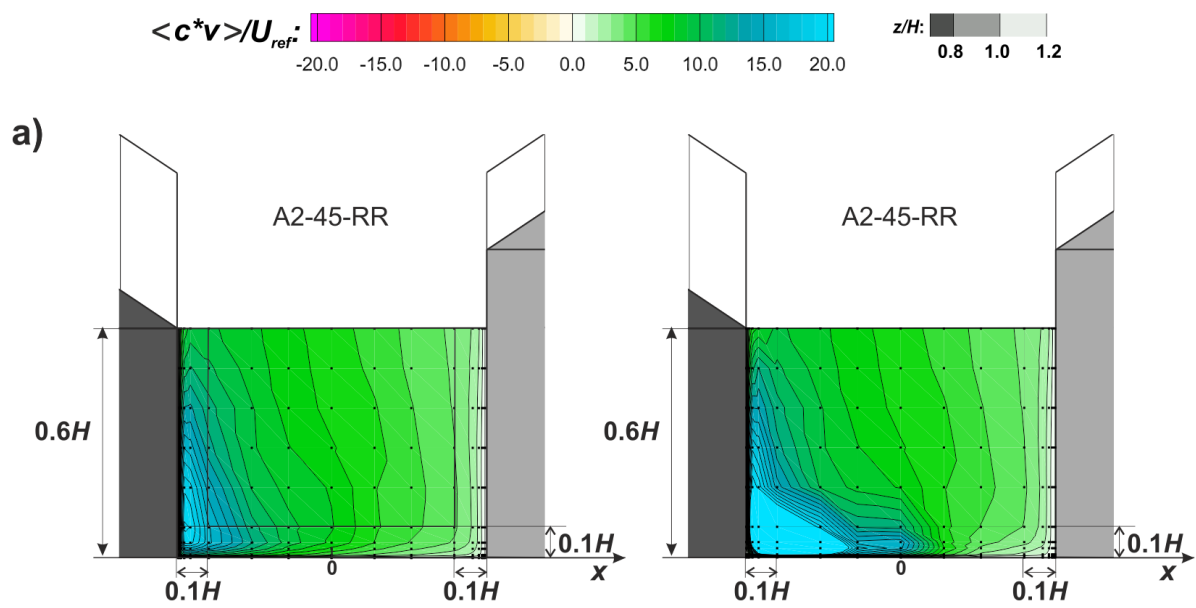

b)

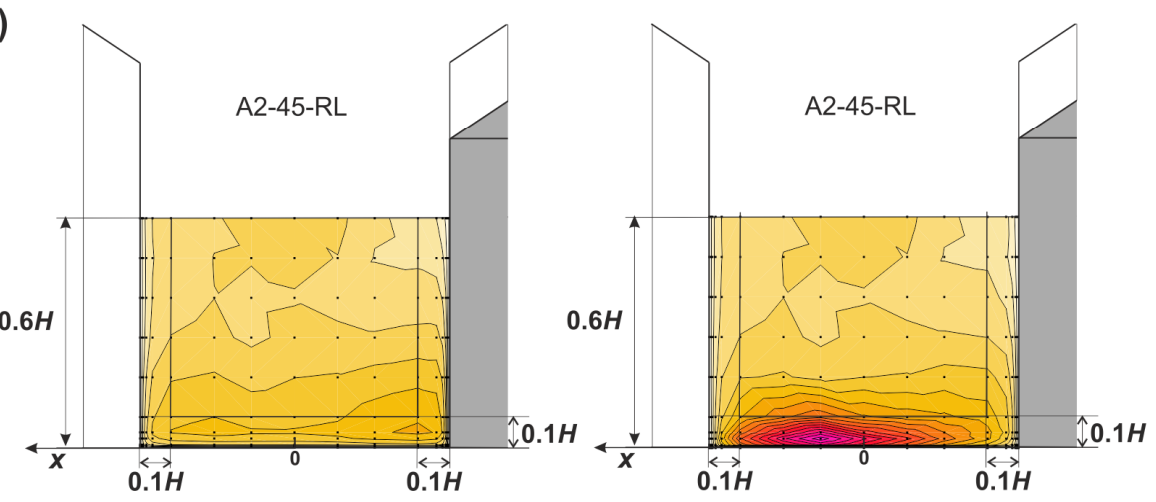

Fig. 7. The same as in Fig. 5, but for the oblique wind (i.e. $45^{\circ}$ ): a) upstream view, b) downstream view. 

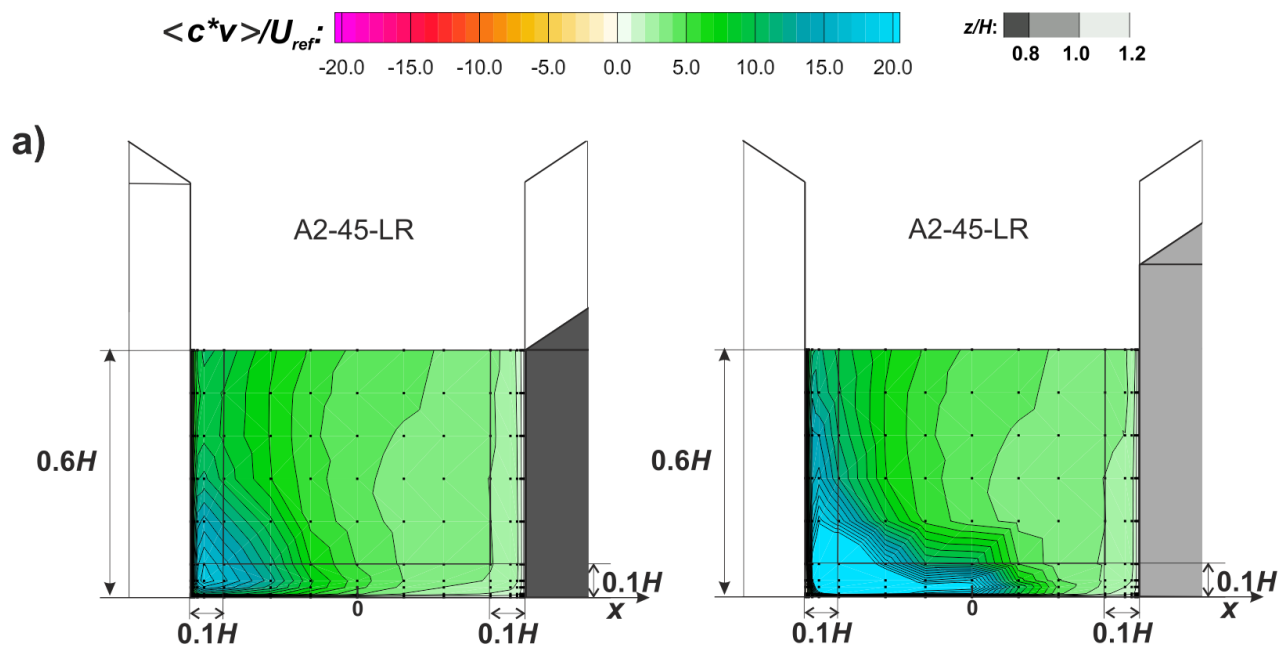

b)

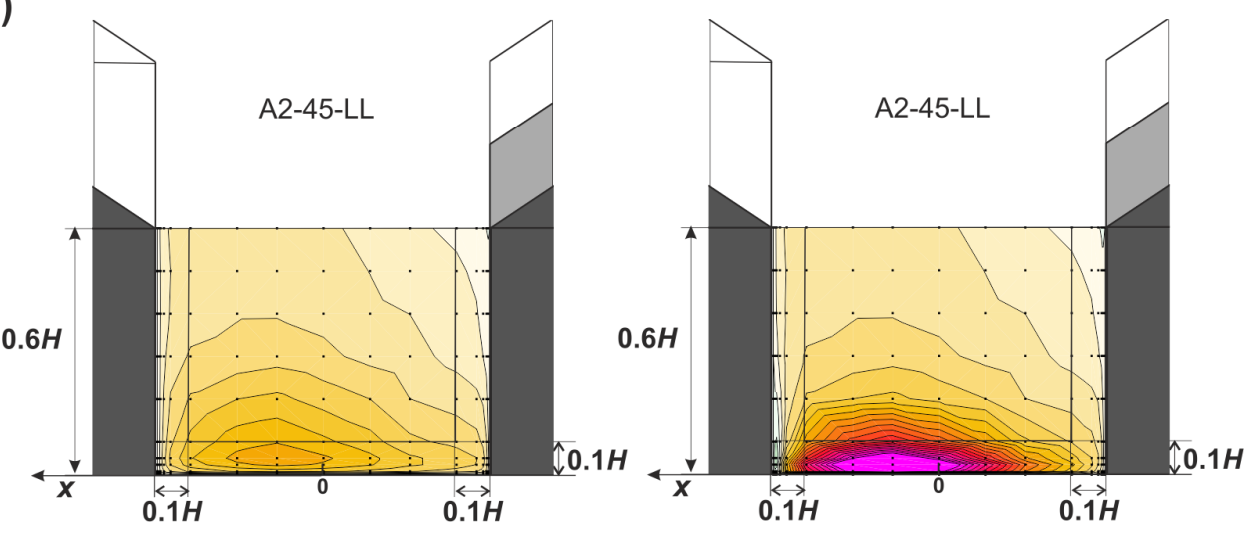

Fig. 8. The same as in Fig. 5, but for the left canyon (L) and the oblique wind: a) upstream view, b) downstream view.

\section{Conclusions}

The aim of this study was to assess the importance of the measurement nearby the street-canyon walls and bottoms for the wind-tunnel experiments. The study was provided by the measurement of the total pollution fluxes through the lateral openings of two different street-canyon models within an urban area with the geometry typical for Central European cities, consisting of pitched roofs of non-uniform height. For the analysis, the two wind directions were chosen (perpendicular and oblique to the along-canyon axis).

It was found that the total pollution fluxes vary significantly nearby the bottoms of the investigated canyons, especially for the oblique wind direction, where the interpolation between the last measured point (at the distance of $0.1 H$ from the border) and the street-canyon bottom almost completely omit the pollution-flux gradients. Thus the bottom line of this study is that the pollution fluxes are needed to be measured at least at the distances of $0.05 \mathrm{H}$ from the walls and bottom in order to properly investigate the street-canyon ventilation (pollution flux balance) through the intersections.

The study was supported by the Charles University, project GA UK No. 1583217.

\section{References}

1. M. Lateb, R. N. Meroney, M. Yataghene, H. Fellouah, F. Sahel, M. C. Boufadel. On the use of numerical modelling for near-field pollutant dispersion in urban environments - A review. Environ. Pollut. 208, 271-758 (2016)

2. H. L. Higson, R. F. Griffiths, C. D. Jones, D. J. Hall. Flow and dispersion around an isolated building. Atmos. Environ. Vo. 30, isme 16, 2859-2870 (1996)

3. J. Shen, Z. Gao, W. Ding, Y. Yu. An investigation on the effect of street morphology to ambient air quality using six real-world cases. Atmos. Environ. Vo. 164, 85-101 (2017)

4. Š. Nosek, L. Kukačka, K. Jurčáková, R. Kellnerová, Z. Jaňour. Impact of roof height non-uniformity on pollutant transport between a street canyon and intersections. Environ. Pollut. 227, 125-138 (2017)

5. M. Carpentieri, P. Hayde, A. G. Robins. Wind tunnel measurements of pollutant turbulent fluxes in urban intersections. Atmos. Environ. 46, 669-679 (2012)

6. W. H. Snyder. Guidelines for fluid modelling of atmospheric diffusion. EPA office of Air quality (1981) 